\title{
Total cross sections for the scattering of low-energy electrons by helium metastable atoms
}

\author{
W G Wilson $\ddagger$ and W L Williams \\ Physics Department, Lniversity of Michigan, Ann Arbor, Michigan 48104, USA \\ Received 10 April 1975, in final form 27 August 1975
}

\begin{abstract}
The absolute total electron scattering cross section for $\mathrm{He}\left(2^{1} \mathrm{~S}_{0}\right)$ and the relative total electron scattering cross section for $\mathrm{He}\left(2^{3} \mathrm{~S}_{1}\right)$ have been measured in the electron energy range $0 \cdot 45-9.4 \mathrm{eV}$. The results show a structureless decrease of the cross sections with increasing electron energy. Comparisons of the results with theory and with the experimental results of Neynaber et al for the triplet scattering cross section are given.
\end{abstract}

\section{Introduction}

Considerable theoretical effort has been devoted to calculating cross sections for the scattering of low-energy electrons by metastable helium atoms. The theoretical results based on the close-coupled approximation (Marriott 1966, Burke et al 1969), the extended-polarization approximation (Sklarew and Callaway 1968) and the variational calculations (Oberoi and Nesbet 1973) predict peaks in the scattering cross sections. These results differ from the predictions using the polarized-core approximation (Husain et al 1967) and from effective potential calculations (Robinson 1969), which yield smooth decreases of the cross sections with increasing electron energy.

Only one previous experiment has been carried out to study low-energy electron scattering by metastable helium. Neynaber et al (1964) measured the absolute total cross section for $\mathrm{He}\left(2^{3} \mathrm{~S}_{1}\right)$ at five electron energies in the range $0.87-8.25 \mathrm{eV}$. We report here the results of experiments to determine the absolute total scattering cross section for low-energy electrons on $\mathrm{He}\left(2{ }^{1} \mathrm{~S}_{0}\right)$ as well as to re-examine the $\operatorname{He}\left(2{ }^{3} \mathrm{~S}_{1}\right)$ cross section, in particular with respect to the presence of structure as a function of electron energy. The experimental details are given in $\$ 2$, the measurements are discussed in $\$ 3$ and $\$ 4$ contains a discussion of the results.

\section{Experimental details}

\subsection{Method}

The cross sections were determined by measuring the reduction in the intensity of a collimated thermal beam of metastable helium atoms when crossed by an electron

+ Research supported in part by the US Atomic Energy Commission. Based on a thesis presented to the Department of Physics, University of Michigan, by W G Wilson in partial fulfilment of the requirements for the $\mathrm{PhD}$ degree.

$\ddagger$ National Science Foundation Predoctoral Fellow. Present address: Fakultät für Physik, Universität Bielefeld. Federal Republic of Germany. 
beam of known energy. The total scattering cross section $Q$ was calculated using the relation

$$
Q=\frac{S h}{I_{\mathrm{e}} I \overline{V^{-1}}} .
$$

Here $S$ is the rate at which the particles are scattered out of the atom beam of intensity $I$ and mean inverse velocity $\overline{V^{-1}}$ by a crossed electron beam of intensity $I_{\mathrm{e}}$ and height $h$. The scattering signals due to the different metastable states in the atom beam were distinguished by quenching the $\mathrm{He}\left(2{ }^{1} \mathrm{~S}_{0}\right)$ component of the metastable beam with $2 \mu \mathrm{m}$ resonance radiation obtained from a helium discharge lamp. This technique, originally developed by Fry and Williams (1969), induces a transition in which an atom in the singlet metastable state $\left(1 \mathrm{~s} 2 \mathrm{~s}, 2{ }^{1} \mathrm{~S}_{0}\right)$ is excited to the $(1 \mathrm{~s} 2 \mathrm{p}$, $\left.2^{1} \mathrm{P}_{1}\right)$ state which in turn decays primarily to the ground state $\left(1 \mathrm{~s}^{2}, 1^{1} \mathrm{~S}_{0}\right)$, thus depopulating the singlet metastable state. The probability for the decay of the $2^{1} \mathrm{P}$ excited state to the $1^{1} \mathrm{~S}$ ground state is more than 1000 times greater than the transition probability for decay back to the $2^{1} \mathrm{~S}$ metastable state. There are, of course, excitation channels open due to other resonance radiation present, but all of these decay mainly via electric dipole radiation to the ground state. The helium atoms in the triplet metastable state $\left(1 \mathrm{~s} 2 \mathrm{~s}, 2{ }^{3} \mathrm{~S}_{1}\right)$ are also excited to higher states, but these decay primarily back to the same state because of the electric dipole selection rule $\Delta s=0$.

As only knowledge of the ratio $S / I$ (see equation (1)) is required for calculation of the cross sections, it was unnecessary to make an absolute measurement of the total flux of atoms in the respective metastable states. In particular, it was not necessary to know the secondary electron emission coefficient for the surface employed to detect the metastable atoms. This ratio could be measured for atoms in the $2^{1} \mathrm{~S}_{0}$ state. The determination of the total singlet scattering cross section therefore comprises an absolute measurement. This ratio could not be determined for atoms in the $2^{3} \mathrm{~S}_{1}$ state. This is because the surface detector was sensitive to resonance radiation in the beam and the content of this radiation could not be measured with the apparatus used for the experiment. Thus the determination of the total triplet scattering cross section comprises a relative measurement.

\subsection{Apparatus}

Figure 1 is a schematic diagram of the apparatus. Metastable helium atoms were produced in a low-voltage, low-pressure gas discharge cell. Any charged particles in the beam were removed by the field of a pair of electrostatic deflection plates. The cell and the deffection plates were in a vacuum chamber maintained at $2 \times 10^{-6}$ Torr.

The source cell was followed by a collimating slit and a $2 \mu \mathrm{m}$ optical quenching region. The electron beam was produced by a gun similar in construction to that described by Collins et al (1970). The electron gun was housed in a vacuum chamber where the pressure was typically $3 \times 10^{-7}$ Torr. The electrode arrangement used for the total cross section measurements is shown in figure 2. A magnetic field of approximately $200 \mathrm{G}$ was used to collimate the electron beam. No grid was used on the exit side of the scattering region to avoid production of secondary electrons.

The energy of the electrons in the interaction region was determined by a retardation method with corrections for contact potential differences and the effects of space charge, the details of which have been given by Collins et al (1970). The contact 


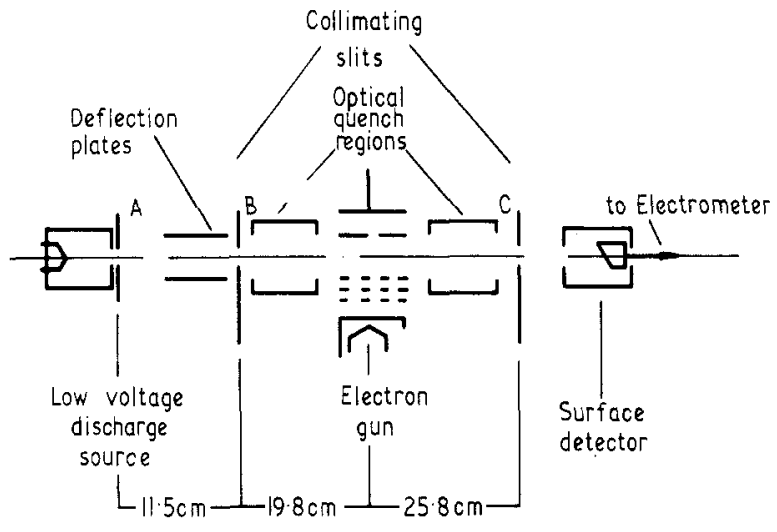

Figure 1. Schematic view of apparatus. The slit dimensions are: A, $0.16 \mathrm{~mm} ; \mathrm{B}, 0.25 \mathrm{~mm}$; C, $1.0 \mathrm{~mm}$.

potential difference between the scattering region and the cathode was found by measuring the change in the observed electron energy distribution as a function of the potential of the scattering region, used here as a filter to remove electrons having energies below a particular value. Figure 3 illustrates an energy calibration sequence. A typical correction to the electron energy for contact potential differences was $(-0.75 \pm 0.15) \mathrm{eV}$. This correction varied negligibly from run to run, but did change over a period of months as a result of contamination of various gun surfaces. The shift in electron energy arising from space charge was calculated by solving Poisson's equation for the potential distribution in the interaction region for the particular geometry and extant charge density. The influence of the field of the anode on the potential distribution was also considered. The total electron current was chosen so that the depression of the potential in the interaction region caused by space charge effects was approximately compensated by the penetration of the anode

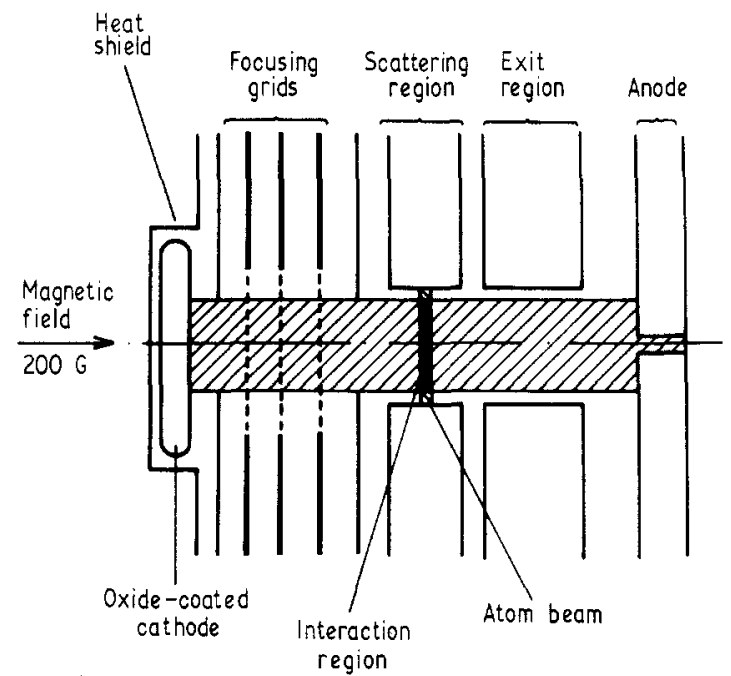

Figure 2. Electron gun. (The exit region is used to reduce the penetration of the anode field into the scattering region.) 


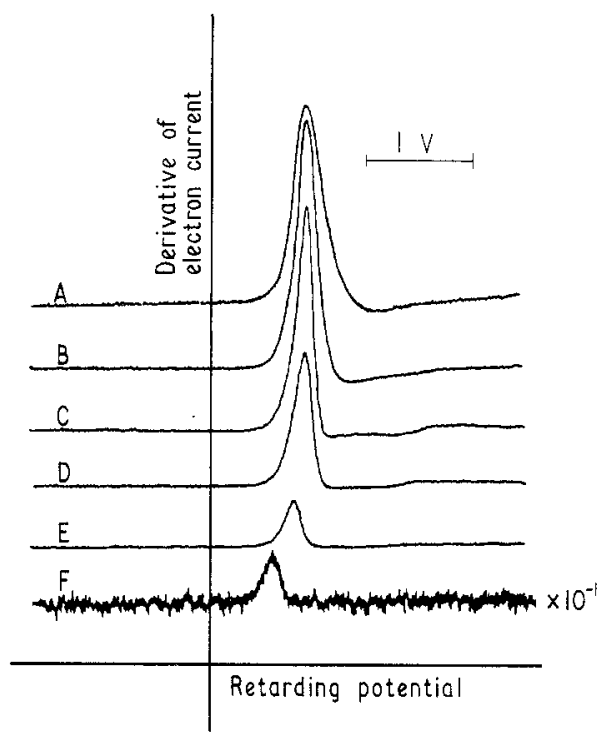

Figure 3. Typical sequence of retarding potential resolution curves with the scattering region potential taken as a parameter. Curve $\mathrm{A}$, scattering region potential $4.0 \mathrm{~V}$; $\mathrm{B}$. $2.0 \mathrm{~V}: \mathrm{C}, 1.0 \mathrm{~V} ; \mathrm{D}, 0.8 \mathrm{~V} ; \mathrm{E}, 0.6 \mathrm{~V} ; \mathrm{F}, 0.4 \mathrm{~V}$

field, yielding the smallest energy spread in the interaction region. The typical correction to the electron energy for space charge effects was $(-0.03 \pm 0.06) \mathrm{eV}$. The corresponding electron current densities were $1-5 \mathrm{~A} \mathrm{~m}^{-2}$. This procedure for calibrating the electron energy was checked by measuring the excitation function of a metastable state in argon. The two methods yielded agreement to $\pm 1.2 \%$.

The electron gun was followed by a second optical quenching region, detector slit and a shielded gold-plated brass surface detector. The latter, in combination with an electrometer, was used to measure the intensity of the metastable atom beam. The second optical quenching region (which is employed for the triplet cross section measurements) removes atoms which have undergone $2^{3} \mathrm{~S}-2{ }^{1} \mathrm{~S}_{0}$ spin-flip scattering, a process that otherwise would not be detected. This detector is also sensitive to photons from the discharge, but not to ground-state atoms. The detector chamber, maintained at $1 \times 10^{-7}$ Torr, was connected to the electron gun chamber by a bellows and rotated about an axis through the centre of the scattering region.

For measurements to determine the velocity distribution a channeltron was used in combination with a mechanical beam chopper. The value of $\overline{V^{-1}}$ calculated from the measured velocity distributions was relatively insensitive to the source conditions. A typical velocity distribution is shown in figure 4. The measured distribution is that of a modified Maxwellian distribution with an effective source temperature of about $540 \mathrm{~K}$.

Adopting the convention proposed by Kusch (1964), the angular resolution of this system can be obtained entirely from the geometry of the apparatus. The angle through which an atom must be scattered to fulfill the '50\% criterion' is about $3 \times 10^{-3} \mathrm{rad}$. This is not significantly modified when the correction for finite beam height is made as the height-to-width ratio of the beam is greater than ten. The effective angular resolution in terms of the electron polar scattering angle, averaged 
over the metastable atom velocity distribution, ranges from $22.2^{\circ}$ at an electron energy of $0.7 \mathrm{eV}$ to $11.4^{\circ}$ at an electron energy of $10 \mathrm{eV}$.

The scattering electron beam was modulated by applying a $73 \mathrm{~Hz}$ square-wave voltage to the first grid of the electron gun. This produced a corresponding modulation of the atom beam of approximately $10^{-4}$ times the total beam flux. Under normal operating conditions this resulted in a modulation current of $0.05 \mathrm{fA}$ RMS with accompanying noise of $10 \mathrm{fA}$ RMS.

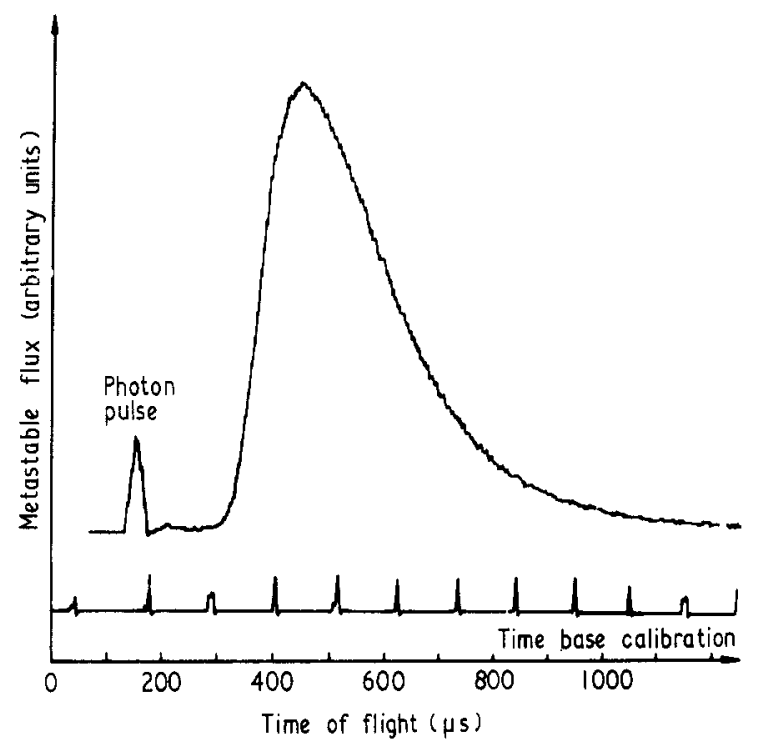

Figure 4. Typical time-of-flight spectrum of metastable helium.

A simplified schematic diagram of the circuit used to simultaneously measure the modulated electron beam flux $I_{\mathrm{e}}$, the total metastable beam flux $I$ and the modulated (scattered) metastable beam flux $S$ is shown in figure 5 . As the same detection system was used to measure $I$ and $S$, and as only the ratio $S / I$ is necessary to calculate the cross sections (see equation (1)), it was not necessary to know either the absolute detector efficiency or the absolute electrometer transfer characteristic. However, the transfer characteristic exhibited a high-frequency roll-off, being down by $3 \mathrm{~dB}$ at $562 \mathrm{~Hz}$. Thus the effective transfer impedance seen by the modulated signal $S$ was less than that for the total (DC) metastable signal $I$. The high-frequency roll-off was determined from measurements of the response of the electrometer when a beam of metastable argon atoms, the intensity of which was modulated with a square wave, was incident on the surface detector. The transfer impedance correction was dependent only upon the measured time constant of the electrometer and the modulation frequency of the electron current and was, therefore, calculable. The signals on the electrometer output from the modulated component of the metastable beam and from the total metastable beam were separated, further amplified and time-averaged. The modulated signal from the electron beam was amplified and also time-averaged. All amplifiers and time-averaging systems were calibrated by measuring their responses to appropriate low level voltages. 


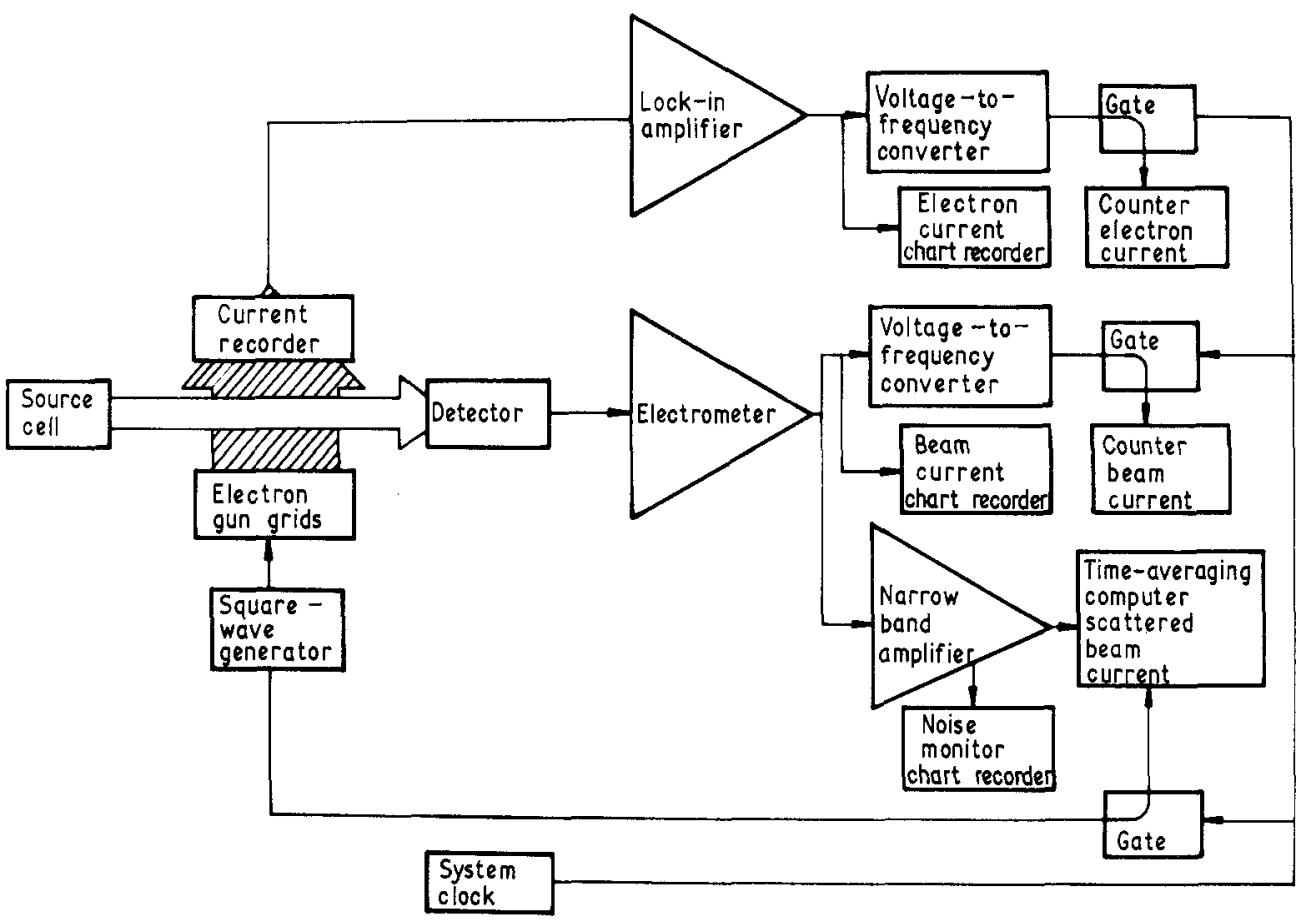

Figure 5. Simplified schematic diagram of the signal averaging circuits.

\subsection{Procedure}

The data needed for calculating the triplet scattering cross section were obtained from measurements made on a totally quenched beam, while the singlet scattering cross section was calculated from measurements on quenched and unquenched beams. Data from two quenched beams and from two unquenched beams were obtained at each energy value. In any run sequence, a measurement at an electron energy value of $7.94 \mathrm{eV}$ was also included. This was done so that the triplet scattering cross section obtained from different measurement sequences could be compared. This energy value was chosen as the normalization point because the signal-to-noise ratio was most favourable here, the cross section varied slowly with electron energy in this region and the only other experimental measurements included a measurement near this value with a minimum in the stated uncertainty.

\section{Results}

\subsection{Data}

Figure 6 shows the measured total electron scattering cross section for helium in the singlet metastable state with the calculation of the elastic scattering cross section of Husain et al (1967). Figure 7 shows the relative electron scattering cross section for helium in the triplet metastable state normalized to $165 \pi a_{0}^{2}$ at an electron energy of $7.94 \mathrm{eV}$. Included are the experimental data of Neynaber et al (1964) and the results of various elastic and total cross section calculations. 


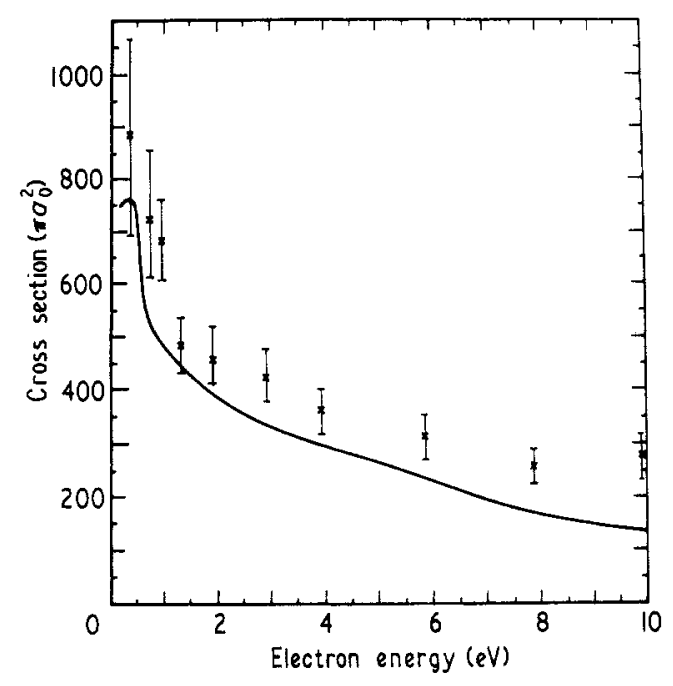

Figure 6. Absolute total electron scattering cross section for helium in the singlet metastable state. $\times$, present work; —. Husain et al (1967).

\subsection{Errors}

The major source of systematic error can be traced to the electron gun. Secondary electrons, produced by primary electrons incident on the various surfaces in the gun, may traverse the scattering region, enhancing the observed scattering signal. To minimize this effect the arode was biased $22.5 \mathrm{~V}$ above the scattering region potential, thus preventing any secondary electrons of lower energy from returning to the

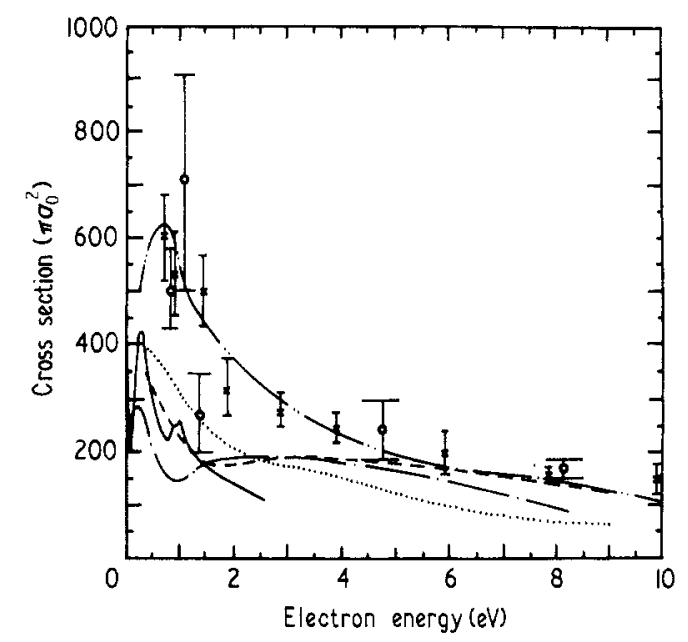

Figure 7. Total electron scattering cross section for helium in the triplet metastable state (present data normalized to $165 \pi a_{0}^{2}$ at $7.94 \mathrm{eV}$ ). $\times$, present work; $O$, Neynaber et al (1964); -.- Burke et al (1969); …., Robinson (1969); -...-. Husain et al (1967); ----, Sklarew and Callaway (1968); _- Oberoi and Nesbet (1973). 
scattering region. Those electrons which collide with lateral surfaces in the gun only produce secondaries at the higher energies. Consideration of worst-case conditions yields a maximum systematic error of $+0.45^{\circ} \%$. Reflection of primary electrons from the gun surfaces can also result in an enhancement of the observed scattering signal. Again, considering worst-case conditions, this results in a maximum error of $+4.9 \%$. The error resulting from an increased scattering path length because of spiralling of the electrons in the magnetic field can be estimated for the various effects which produce transverse velocity components for the electrons in the interaction region. The major contributor to this source of error is the thermal emission velocity of the electrons from the cathode. The resulting uncertainties are $\pm 10.5 \%$ at the lowest energy and $\pm 0.47 \%$ at the highest energy. The focusing effect of the grid elements contributes $\pm 1.3 \%$ and $\pm 0.01 \%$, respectively, for these energies. Other sources of electron spiralling are negligible.

The uncertainties in the measurement of the velocity distribution of the metastable atoms and calculation of $\overline{V^{-1}}$ result in an uncertainty of $\pm 6.8 \%$.

The smallest identifiable source of systematic error was due to drifts in the metastable beam flux, which ranged from $(-0 \cdot 1)-(+2 \cdot 3) \%$, depending on the beam considered. Some drift was always observed, but this was minimized through the separate control of the source cell discharge voltage and discharge current. Because the beam drift was found to be approximately linear in any given time period, it was possible to remove all dependence of the singlet measurement on beam drift. The measurement of the total singlet scattering cross section does not depend upon the efficiency of the quench region and adds a negligible systematic error to the total triplet scattering cross section. All other sources of possible systematic error such as "scattering in" signal due to beam width and corrections due to the finite detector size (non-observation of small-angle scattering) only contributed a maximum of $\pm 2.1 \%$ error.

Possible systematic errors associated with the applicability of equation (1) remain to be discussed. The derivation of this equation has been considered in detail elsewhere (Rubin et al 1969). Involved in the use of this expression is the assumption that the dependence of the cross section of the relative velocity of the electron and metastable atom could be ignored. Estimates of the uncertainty resulting from this assumption yield $\pm 0.78 \%$. The evaluation of the overlap integral is usually very difficult for non-uniform crossed beams. However, if either beam is uniform, the evaluation is straightforward. Uniformity of the metastable beam in the interaction region need only be assumed in the direction parallel to the atom beam and in the direction mutually perpendicular to the electron and atom beams. The first assumption is justifiable in that only approximately one in $10^{4}$ atoms are removed from the beam in the scattering process. The second assumption is justifiable on the basis of the fact that the metastable beam is produced with an effusive source and the interaction region itself acts as a collimating slit in this direction, i.e. there is no penumbra in the interaction region in this direction. There is a small contribution from the angular spread of the metastable beam, resulting in an error of less than $0.1 \%$. As no assumptions have been made concerning the electron beam uniformity, the overlap integral will be the same for all electron energies considered. Assuming all of the error sources considered were independent, the total systematic error in the measurement of the total cross section ranged from $(-12 \cdot 9)-(+14.0) \%$ at the lowest electron energy to $(-7 \cdot 5)-(+9 \cdot 2) \%$ at the highest electron energy. The statistical errors in the final data range from $\pm 21.6 \%$ to $\pm 10.3 \%$ over the same range of electron energy. The absolute energy of the incident electrons is known to $\pm 0.15 \mathrm{eV}$. 


\section{Discussion of results}

The $2^{1} \mathrm{~S}$ metastable state of helium is $20.61 \mathrm{eV}$ above ground state. Immediately below this state is the $2^{3} \mathrm{~S}$ metastable state at $19.82 \mathrm{eV}$ and above it the $2^{3} \mathrm{P}, 2^{1} \mathrm{P}$ and $3^{3} \mathrm{~S}$ states at $20.91,21.20$ and $22.65 \mathrm{eV}$, respectively. The ionization potential of helium is at $24.47 \mathrm{eV}\left(3.86 \mathrm{eV}\right.$ above the $2^{1} \mathrm{~S}$ metastable state). The threshold for inelastic scattering processes from the metastable singlet state appears at electron energies above $0.30 \mathrm{eV}$ while superelastic scattering of electrons can occur at all energies. The data presented here show a rapid decrease from $(883 \pm 191) \pi a_{0}^{2}$ at $0.45 \mathrm{eV}$ to $(483 \pm 53) \pi a_{0}^{2}$ at $1.47 \mathrm{eV}$. From this point the fall is less rapid with increasing electron energy and falls to $(278 \pm 43) \pi a_{0}^{2}$ at $9.94 \mathrm{eV}$. The data are consistently higher than the elastic electron scattering cross section calculation of Husain et al (1967), although the error bars in the low-energy region do overlap this calculation. This is as expected since at higher electron energies, scattering processes other than elastic scattering may occur and contribute to the total scattering cross section. It is to be noted that the results of Husain et al (1967) are only applicable to the 'gross structure' of the cross section. No resolved low-energy peak in the total electron scattering cross section is present. The total electron scattering cross section data from the triplet metastable state can occur at electron energies above $0.79 \mathrm{eV}$, while again superelastic scattering may occur at all energies. Here the total scattering cross section falls rapidly from $(3.66 \pm 0.45) \times Q(7.94 \mathrm{eV})$ at $0.70 \mathrm{eV}$ to $(1.97 \pm 0.40)$ $\times Q(7.94 \mathrm{eV})$ at $9.94 \mathrm{eV}$.

The triplet data follow the same general shape obtained by Neynaber et al (1964) except that the peaking of the electron scattering cross section conjectured by them at low electron energies was not resolved. Some of the data points, when normalized to Neynaber et al (1964) at approximately $8 \mathrm{eV}$, fall below the elastic scattering cross section obtained by Husain et al (1967). If, instead, the data are normalized to the value obtained by Neynaber et al (1964) at approximately $5 \mathrm{eV}$, then this apparent discrepancy is removed. (It can also be removed by using a value for the cross section at approximately $8 \mathrm{eV}$ that is larger, but still within the stated experimental error.) All of the other theoretical calculations of the elastic scattering cross section are well below the data presented here, when normalized as indicated. The only total electron scattering cross section calculations predict two peaks, one near $0.2 \mathrm{eV}$ and the other near $1.5 \mathrm{eV}$. Because of the experimental error herein, the presence of these peaks could not be confirmed. Both of these results were, however, a factor of 2-3 below the triplet data of Neynaber et al (1964).

\section{Acknowledgments}

We are grateful to the US Atomic Energy Commission for their financial support, and to Mr R E Collins for sending us the machine drawings of the NYU electron gun. We wish to thank Mr D Walden for his technical assistance and encouragement.

\section{References}

Burke P G, Cooper J W and Ormonde S 1969 Phys. Rev. 183245

Collins R E, Aubrey B B, Eisner P N and Celotta R J 1970 Rer. Sci. Instrum. 411403 
Fry E S and Williams W L 1969 Rer. Sci. Instrum 401141

Husain D, Choudhury A L, Rafiqullah A K, Nestor C W Jr and Malik F B 1967 Phys. Ret. 16168 Kusch P 1964 J. Chem. Phys. 401

Marriott R 1966 Proc. Phys. Soc. 87407

Neynaber R H, Trujillo S M, Marino L L and Rothe E W 1964 Proc. 3rd Int. Conf. on Physics of Electronic and Atomic Collisions (Amsterdam: North-Holland) p 1089

Oberoi R S and Nesbet R K 1973 Phys. Ret. A 82969

Robinson E J 1969 Phys. Rev. 182196

Rubin K, Bederson B, Goldstein M and Collins R E 1969 Phys. Ret. 182201

Sklarew R C and Callaway J 1968 Phys. Rev. 175103 